\title{
Extraction Of Reaction Amplitudes From Complete Photo-Production Experiments
}

\author{
A.M. Sandorfia and S. Hoblit ${ }^{\mathrm{b}}$ \\ ${ }^{a}$ Physics Dept., Thomas Jefferson National Accelerator Laboratory, Newport News, VA 23606 USA. \\ ${ }^{b}$ National Nuclear Data Center, Brookhaven National Laboratory, Upton, NY 11973 USA
}

\begin{abstract}
A new generation of complete experiments in pseudoscalar meson photo-production is being pursued at several laboratories. Here the designation of complete refers to measurements of most if not all of the possible reaction observables, of which there are 16 involving spins of the beam, target and recoil baryon. Hyperon production to $\Lambda$ or $\Sigma^{+}$final states affords attractive opportunities, since their weak decays provide an efficient self-analysis of their spin. When the beam and target are also polarized, the resulting triple polarization measurements determine the full suite of observables with a single target orientation. This has been a focus at Jefferson Lab in the recently completed g9/FROST and g14/HDice experiments now under analysis. Multipole analyses of $\gamma p \rightarrow \mathrm{K}^{+} \Lambda$ have been carried out with recently published (incomplete) polarization data, and the uniqueness of the extracted amplitudes has been studied. Experiments with realistically achievable uncertainties require a significantly larger number of spin asymmetries than the in-principle minimum needed for a mathematical solution of the amplitude.
\end{abstract}

Keywords: meson photo-production, baryon resonances, multipole analysis, polarization. PACS: 13.60Le, 13.75.Gx, 13.75.Jz, 13.88.+e, 14.20.Gk;

\section{THE N* PROBLEM AND MEASUREMENTS OF EVERYTHING}

The low-energy structure of QCD lies encoded in the excited-state spectrum of the nucleon, which is a complicated overlap of many resonances. Recently, Lattice QCD calculations [1] have confirmed the long standing Quark Model predictions [2] of a large number of excited nucleon states, only a fraction of which have been identified. Only the lowest predicted levels have been reported and these come mainly from analyses of $\pi \mathrm{N}$ scattering. This may be a reflection of the $\pi \mathrm{N}$ partial widths, which are predicted to diminish with energy while higher lying states are expected to have larger branches to KY and $\pi \pi N$ final states. Such final states are excited in photo-production and the spin of the photon provides additional probes of the amplitude. The outstanding challenge is to unravel the $\mathrm{N}^{*}$ spectrum in as model-independent a way as possible. The goal of a recent series of experiments at Jefferson Lab is a measurement of all possible spin-observables in order to determine the production amplitude as a curve in the complex plane. This would serve as the ultimate starting point in a search for poles by analytic continuation. Since the electromagnetic interaction does not conserve isospin, this requires data on both proton and neutron targets. The experiments with polarized protons were the focus of the g9/FROST runs, while the g14/HDice experiment focused on polarized neutron reactions. 
In single-pseudoscalar meson photoproduction there are 16 possible observables, the cross section $(\sigma)$, three asymmetries which to leading order enter the general cross section scaled by a single polarization of either beam, target or recoil $(\Sigma, T, P)$, and three sets of four asymmetries whose leading dependence in the general cross section involves two polarizations of either beam-target $(E, G, F, H)$, beam-recoil $\left(C_{x}, C_{z}\right.$, $O_{x}$, $\left.O_{z^{\prime}}\right)$ or target-recoil $\left(L_{x}, L_{z} ; T_{x}, T_{z^{\prime}}\right)$. However, each of these observables enters the general form of the cross section scaled by two terms containing different combinations of beam, target and recoil spins [3]. This leads to two distinct ways to measure each observable.

There is some confusion in the literature regarding definitions of asymmetries and the conventions used in partial wave analyses (PWA). This arises from the use of different coordinate systems and because the same symbol or asymmetry name has been used by different authors to refer to different experimental quantities. Expressions for the photo-production asymmetries as coordinate-system independent ratios of cross sections, along with the names used for these ratios by different PWA groups, can be found in ref [4]. Examples of the explicit measurements needed to construct all of the observables are given in the appendices to ref [3].

In pseudoscalar meson photo-production, the beam, target and recoil baryon can all be polarized, although these polarizations are not all on an identical footing. While the beam and target polarization are under experimental control, the recoil polarization is rather a byproduct of the entrance channel angular momentum and the reaction physics. As such, the recoil polarization can be written in terms of various combinations of different spin asymmetries [3]. This can lead to a particularly fruitful arena in which different components of the recoiling baryon's polarization determine various spin observables. The two most practical reactions for this are in KY production, where the angular distribution in the weak decay of the hyperon provides a self-analysis of the polarization,

$$
\begin{aligned}
& \gamma \mathrm{N} \rightarrow \mathrm{K} \Lambda \Rightarrow \mathrm{K}\left(\pi^{-} \mathrm{p}\right), \\
& \gamma \mathrm{N} \rightarrow \mathrm{K} \Sigma^{+} \Rightarrow \mathrm{K}\left(\pi^{0} \mathrm{p}\right) .
\end{aligned}
$$

The $\mathrm{K} \Lambda$ channel has an analyzing power of $2 / 3$ while that of the $\mathrm{K} \Sigma^{+}$channel is almost unity. When a quasi- $4 \pi$ detector is used, such as the CLAS at Jeffferson Lab, such recoil polarization information is in the data stream and comes without a significant statistical penalty.

Each of the 16 spin observables can be written in terms of 4 complex amplitudes, which can be expressed in ether Cartesian $\left(F_{i}\right)$ [5], Spherical or Helicity $\left(H_{i}\right)$, or Transversity $\left(b_{i}\right)$ representations [6]. An explicit decomposition of all 16 into the 4 CGLN $F_{i}$ amplitudes is given in [3]. However, the four amplitudes in each of these representations are angle dependent. Extracting them directly from experiment would require separate fits at each angle, which greatly limits the data that can be used and requires some model-dependent scheme to constrain an arbitrary phase that could be angle-dependent. The solution to this intractable situation is a Wigner-Echart style factorization into reduced matrix elements, multipoles, and simple angle-dependent coefficients from angular momentum algebra $[3,5]$. One can then fit the multipoles 
directly, which both facilitates the search for resonance behavior and allows the use of full angular distribution data at a fixed energy to constrain angle-independent quantities. The price is a significant increase in the number of fitting parameters, but since the excited states of the nucleon are associated with discrete values for their angular momentum, this expansion of variables is inevitable.

Since the full amplitude is a sum of four independent complex terms, measurements of eight different observables are needed for a mathematical solution. (In practice, given realistic uncertainties on data, many more are required. But more on that later.) Since there are a total of 16 spin observables, it is clear that they are not all independent. A set of 37 Fierz Identities interrelate the spin asymmetries [3,7]. They provide important constraints on the self-consistency of data used in a multipole fit, once a sufficiently large set of asymmetry measurements is available. The first 2 to be applied with recently published $\gamma \mathrm{p} \rightarrow \mathrm{K}^{+} \Lambda$ data are $[3,8]$,

$$
\begin{array}{r}
\Sigma P-C_{x^{\prime}} O_{z^{\prime}}+C_{z^{\prime}} O_{x^{\prime}}-T=0 \\
O_{x^{\prime}}^{2}+O_{z^{\prime}}^{2}+C_{x^{\prime}}^{2}+C_{z^{\prime}}^{2}+\Sigma^{2}-T^{2}+P^{2}-1=0
\end{array}
$$

As more polarization data become available, a growing number of Fierz identities can be used to almost eliminate systematic scale shifts between data sets. However, utilizing them requires data sets at common angles and energies, which in practice requires some interpolation.

Two groups of experiments have been carried out at Jefferson Lab that, once analysis has been completed, should provide information on all 16 observables for the $\mathrm{K} \Lambda$ channel and most for $\mathrm{K} \Sigma^{+}$production. Results for $\gamma \mathrm{p} \rightarrow \mathrm{K}^{+} \Lambda$ from the $g l c$ and gl 1 a running periods with liquid hydrogen have already provided the cross section, $P$, $C_{x}$, and $C_{z}$, $[9,10]$. The inclusion of these in the Bonn-Gatchina PWA has lead to possible candidates for new states [11], although the solutions are still ambiguous. The g8 period ran with linear photon polarization from coherent bremsstrahlung to measure $\Sigma, T, P, O_{x}$, and $O_{z}$, and these results are nearly finalized. The $g 9$ periods ran with FROST, a butanol $\left(\mathrm{C}_{4} \mathrm{H}_{9} \mathrm{OH}\right)$ target in which the free $\mathrm{H}$ nuclei were polarized. The $g 9 a$ period held the target spin longitudinally along the beam axis, while in the $g 9 b$ period the target spin was oriented transverse to the beam. Both should be capable of yielding a complete set of asymmetries and the cross comparison between them will provide an important handle on systematics. Most recently, the g14 period completed a run with a polarized solid deuterium-hydride (HD) target, focusing on neutron reactions in polarized deuterium. Once the analyses of all of these measurements are complete, the results are expected to have dramatic impacts on deduced multipoles and on our understanding of the $\mathrm{N}^{*}$ spectrum.

\section{THE UNIQUENESS OF AMPLITUDE EXTRACTIONS}

A critical issue for the goal of determining an amplitude is the uniqueness of the solution. The reaction channel with the largest number of different published observables is $\gamma \mathrm{p} \rightarrow \mathrm{K}^{+} \Lambda$, where results from GLAS glc and $g 11$ [9,10] and from 
GRAAL [8] can be combined to provide values for $\sigma, \Sigma, T, P, O_{x}, O_{z}, C_{x}$, and $C_{z}$, over at least a limited $\mathrm{W}$ range (1676 to $1883 \mathrm{MeV})$. We have carried out a multipole analysis for this combined set using a Monte Carlo sampling of the parameter space, coupled with gradient minimization [3]. These CLAS and GRAAL results determine bands of solutions, that are indistinguishable in their ability to reproduce the data but nonetheless derive from very different multipole amplitudes. The bands represent a shallow $\chi^{2}$ valley pitted with a large number of local minima that are experimentally degenerate. Evidently much more information will be required.

Amplitudes can of course be rotated by an arbitrary phase. There are two methods to remove such ambiguities. The simplest is to set the phase of one particular multipole to zero, which should be a relatively large one (eg. the $\left.E_{0+}\right)$. This does not generally limit the utility of a solution. To compare with results from other PWA one simply rotates all solutions to the same reference phase. (eg. Multiply all multipoles of another solution by $e^{-i \phi}$, where $\phi$ is the phase of the $E_{0+}$ in that solution.) Another method of fixing the overall phase that has been successfully used in charged $\pi$ production is to set high L multipoles to their real Born and $t$-channel pole values [12]. The $t$-channel graphs have significant well-established contributions that extent to very high $L$ and these effectively fix the phase. Unfortunately, in $\mathrm{K}^{+}$production this procedure isn't directly applicable since the $t$-channel couplings are much smaller (being inversely proportional to the mass of the exchanged meson), there are more meson candidates that can be exchanged that are close in mass $\left(\mathrm{K}+(493), \mathrm{K}^{*}(892)\right.$, $\mathrm{K}_{1}(1270)$ and $\mathrm{K}_{1}(1400)$ ) and their couplings are not well known. Thus, for the hyperon channel with the greatest potential to supply a complete set of observables, one will always have to fix the phase of one particular multipole.

We have simulated the ultimate data sets we expect to have available in studies of pseudo-data [3]. Asymmetries were generated from a reference multipole solution at the angles and energies of the CLAS measurements, and then dithered about those points by smearing with Gaussians of width given by the expected experimental errors. Multiple local minima are always present, arising essentially from experimental uncertainties. Nonetheless, very significant gains in narrowing of the solution bands come from the addition of new spin observables. Often, even modest errors on an asymmetry are sufficient to derive a substantial gain. It is clear that the criteria for a mathematical solution that have been discussed in the literature [7] are unfortunately broken by measurement errors in realistic experiments. Nonetheless, studies with mock-data on a complete suite of 16 observables, accompanied by the uncertainties expected from the recently completed measurements, does result in a dramatic narrowing of the multipole uncertainty bands.

We close this short summary with one additional point of caution. In our simulation studies, the positions of mock data points were picked randomly and a band of solutions was found. Choosing another set of randomly smeared data points is equivalent to repeating an experiment. This results in a different band of solutions. The particular allowed solution set depends on the statistical distribution of the data. If we repeat this exercise hundreds of times we map out a distribution of solution bands that is on average about a factor of two wider than any one band derive from a single set of data. This indicates a procedure that will have to be used to expose the full uncertainty on the multipoles extracted from complete experiments. 
- the data from the cross section and as many asymmetries as possible must be combined to map out a solution band;

- one solution near the centroid of this band is used to generate a mock data set of all observables that were measured;

- the actual statistical errors from the experiments define Gaussians that are used to smear the mock data; this mock data set is then used to fit out another solution band; this process is repeated, probably several hundred times.

- the standard deviation of the spread in the solutions from these mock data give the best estimate on the errors of the multipoles deduced from the actual experiment.

While the amplitude extraction will be a somewhat laborious process, it is expected to provide well-defined multipoles which should create a watershed in our understanding of the nucleon spectrum.

\section{ACKNOWLEDGMENTS}

This work was supported by the US Department of Energy, Office of Nuclear Physics Division, under contract DE-AC05-06OR23177 under which Jefferson Science Associates operate Jefferson Laboratory, and also US Department of Energy contracts DE-AC02-98CH10886.

\section{REFERENCES}

1. R. Edwards, J. Dudek, D. Richards and S. Wallace, Phys. Rev. D84, 074508 (2011).

1. S. Capstick and W. Roberts, Phys. Rev. D58, 074011 (98).

2. A.M. Sandorfi, S. Hoblit, H. Kamano and T-S.H. Lee, J Phys G38, 053001 (2011).

3. A.M. Sandorfi, B. Dey, A. Sarantsev, L. Tiator and R. Workman, arXiv:1108.5411v2.

5. G. Chew, M. Goldberger, F. Low and Y. Nambu, Phys. Rev. 105 (1957) 1345.

6. I. Barker, A. Donnachie and J. Storrow, Nucl. Phys. B95 (1975) 347.

7. W-T. Chaing and F. Tabakin, Phys Rev C55 (1997) 2054.

8. GRAAL Collaboration, A. Lleres et al., Eur. Phys. J. A39, 149 (2009).

9. CLAS Collaboration, R.K. Bradford et al., Phys. Rev. C73 (2006) 035202; ibid, C75 (2007) 035205.

10. CLAS Collaboration, M. McCracken et al., Phys. Rev. C81 (2010) 025201.

11. A.V. Anisovich, R. Beck, E. Klempt, V.A. Nikonov, A.V. Sarantsev and U. Thoma, Eur. Phys. J. A48, 14(2012).

12. R.L. Workman, priv. comm.. 